\title{
Méchanceté autofictionnelle dans Folle de Nelly Arcan
}

\author{
Rosemarie Savignac \\ Université du Québec à Montréal
}

Un homme amer publie sur Internet les photos sexuellement explicites que son ancienne flamme lui a envoyées. C'est la revenge porn ou la nouvelle façon de se venger de ceux qui nous ont quittés. Dévoiler l'autre au monde entier, par les réseaux sociaux, dans sa plus complète vulnérabilité. Plusieurs pays ont déjà adopté des lois visant à sanctionner ce phénomène. Qu'en est-il de la littérature ? Nelly Arcan, après avoir été quittée par un homme, dissèque dans un récit autofictionnel les tares, les obsessions, les fétiches sexuels de son ancien amant. S'il n'y a aucune image imprimée dans le récit, la revanche de Nelly Arcan met pourtant littéralement un être à nu. Les secrets 
sordides divulgués correspondent-ils à la réalité ? Qu'en a vraiment à faire le lecteur, sauf s'il s'agit de l'amant d'Arcan luimême, qui pourrait la poursuivre pour atteinte à la vie privée ? Également, l'auteure n'hésite pas dans son récit à divulguer ses propres dépendances. Elle y semble encore plus méchante et dure envers elle-même qu'envers quiconque. Infériorisée par son amant, ridiculisée dans sa peine d'amour, Arcan ne s'épargne pas elle-même. Nous analyserons donc comment la méchanceté littéraire, et plus particulièrement autofictionnelle, possède une autonomie face à la «réalité ». De plus, nous examinerons comment l'ironie caustique et la méchanceté d'Arcan sont utilisées comme des outils langagiers et stylistiques audacieux afin de renverser les inégalités au sein du couple hétérosexuel.

\section{Méchanceté autofictionnelle : implications réelles}

À l'automne 2006, deux ans après la publication de Folle, Nicolas Ritoux, qui a inspiré le personnage de l'amant français à l'écrivaine Nelly Arcan, répond à son ancienne amie de cœur afin de rectifier certains faits dans le magazine Urbania, publié à Montréal. Il écrit : "Contrairement à toi, je ne tire aucun plaisir à faire publiquement de la peine [aux autres]. » (2006, p. 31) Selon lui, Nelly Arcan, dans Folle, a exagéré certaines anecdotes, en a inventées d'autres, pour le faire paraître très mal. Là est le potentiel de méchanceté de l'autofiction: utiliser comme personnages de fiction des personnes réelles (de façon anonyme dans le cas d'Arcan, mais avec leur profession, leur nationalité, leurs relations, bref, tout ce qui permet de les identifier) et dire à leur propos la vérité ou non. 
Commençons donc par circonscrire les implications littéraires de la méchanceté autofictionnelle dans Folle. Dans l'article «Autofiction et roman autobiographique : incidents de frontière ", Yves Baudelle perçoit l'autofiction comme une stratégie éditoriale de controverse : «À l'évidence, l'hybridité $\mathrm{du}$ genre à la fois auto(biographique) et fiction permet de profiter de la demande soutenue du public pour toutes les formes de confession. » (2007, p.52-53) À cet égard, la thématique érotique répond bien à cette avidité du public pour le sensationnalisme. Elle permet de provoquer la curiosité ou l'indignation chez le lectorat. Ce thème, récurrent dans le deuxième livre d'Arcan, incite effectivement le lecteur à questionner la part de vérité dans l'autofiction avec une curiosité pernicieuse. Dans Folle, on tend à réduire à zéro l'espace de fiction, et ce, malgré l'appellation générique « récit » donnée au texte par les Éditions du Seuil. La narratricepersonnage du livre s'appelle Nelly Arcan et a déjà écrit un roman à succès intitulé Putain, à l'instar de l'auteure de Folle, Nelly Arcan, jeune écrivaine à succès. Devant un texte vraisemblablement tout à la fois factuel et fictionnel, le lecteur ne peut consentir qu'à un seul pacte de lecture: celui de l'autobiographie ou celui de la fiction. Dans cette perspective, Simon Harel croira que l'autofiction est une habile contrefaçon. Il écrit, dans Attention écrivains méchants : "On fera valoir que le "réel" de la fiction n'a pas à rendre de comptes à la réalité, que le discours possède son autonomie, son invention face aux réglementations diverses de la réalité. » (2010, p. 49) Harel oppose ici le « réel » à la "réalité », distinction épineuse s'il en est. Le « réel » de la fiction ne s'encombre pas de la réalité des opinions, des polémiques et des rumeurs publiques. Par l'appellation générique «roman» ou «récit», l'auteur peut 
ainsi se distancier des lois et des jugements juridiques ${ }^{1}$. Évidemment, le journaliste français s'est retrouvé dans les traits du personnage d'Arcan; certaines de ses relations se sont également reconnues dans les pages du livre (Ritoux, 2006, p. 30-31). Cependant, les longs épisodes romancés de la relation amoureuse témoignent bien, selon Ritoux, de l'appartenance du livre d'Arcan au genre fictif. Il nie plusieurs éléments du récit publié par son ancienne amoureuse : «Je ne t’ai jamais giflée ou crachée [sic] dessus en baisant. Je t'ai attachée une fois à ta demande et avec la corde que tu m'as fournie. » (Ritoux, 2006, p. 31) C'est entre la parole d'Arcan et celle de Ritoux que le lecteur se voit forcé de choisir la « vraie vérité ».

Si l'autofiction interroge la frontière entre le réel et la réalité, elle rend également poreuses les frontières entre le privé et le public, entre l'intime et l'« extime». Madeleine Ouellette-Michalska, dans Autofiction et dévoilement de soi (2007, p.21), voit la confusion des territoires intimes et collectifs comme une des principales caractéristiques de l'ère postmoderne. Cette particularité s'applique bien à Folle. Le récit appartient au genre épistolier: la narratrice-personnageauteure écrit une lettre très intime à son ancien amant. En publiant cette lettre, Arcan fusionne alors les espaces public et privé. La publication de la lettre et sa circulation physique en tant que livre ont donc la fonction d'une prise de parole publique.

1 Précisons néanmoins que le genre fictionnel ne suffit pas à éviter toutes poursuites. Par exemple, Christine Angot a quant à elle été condamnée pour atteinte à la vie privée en 2013 pour son livre Les Petits. 


\section{Outils langagiers et stylistiques de la méchanceté}

Dans la prose d'Arcan se détectent indéniablement une ironie vindicative ainsi qu'un éventail d'outils langagiers et stylistiques ironiques. Cette méchanceté caustique n'échappe pas à la principale tête de Turc du récit, soit l'amant français : « [T] a manière de me décrire en train de jouer les artistes au café en tentant de devenir l'énième [sic] émulateur de Céline est aussi blessante que vraie (il n'y a que la vérité qui blesse).» (Ritoux, 2006, p. 31) Dans l'ouvrage Le carquois de velours, le postulat de Lucie Joubert est clair: "[L'ironie] permet aux femmes, traditionnellement "objets" d'ironie, de renverser les règles du jeu et de devenir "sujets" ironisants. » $(1998$, p. 18) Ce renversement des rôles est manifeste dans Folle : Arcan devient sujet de son propre récit, transformant l'homme dominant en objet. Par exemple, Arcan écrit : «Ton écriture ne m’intéressait pas, mais toi, si. Ta beauté justifiait tout ce que tu écrivais. [...] De toute façon tout ce que tu faisais se faisait dans le cadre de ta beauté, tu étais comme le DJ Freak, dans ta virilité il y avait l'attrait de formes féminines. » (2004, p. 168-169) On assiste ici au renversement des valeurs masculines et féminines. La beauté de l'homme prend le pas sur son travail créateur, justifie même ses aspirations professionnelles. Alors que la beauté est plutôt une valeur traditionnellement féminine qui tend à objectiver les femmes, en faire la caractéristique première de l'amant renverse les codes des genres ou du moins les rend absurdes. L'expression «formes féminines» associée à «ta virilité » est une antithèse sémantique qui oppose également la rondeur des courbes féminines aux angles des mâchoires, des épaules et des hanches masculines. 
De plus, pour attaquer son ancien amoureux, Nelly Arcan pratique aussi ce que Joubert a appelé le «transfert illocutoire ironique» :

[L]es auteures vont prêter à certains personnages masculins des paroles sexistes, misogynes ou simplement paternalistes pour dénoncer, à travers ces boucs émissaires, les attitudes de mépris, de condescendance ou de supériorité qu'adoptent quelquefois les hommes à l'égard des femmes. (Joubert, 1998, p. 54)

L'amant français est le bouc émissaire par excellence. Il représente l'Autre dans sa plus grande altérité : l'homme, le Français, le journaliste. Son discours, misogyne et dégoulinant de condescendance, est dénoncé par l'esprit critique d'Arcan dans Folle :

Tu m'as dit que si on cherchait à couvrir de sperme le visage des femmes dans les films pornos, c'était parce qu'elles méritaient une bonne leçon et non parce qu'elles exigeaient des hommes la preuve matérielle de leur pouvoir de séduction. Selon toi, salir ne voulait pas dire rassurer, ça voulait dire rejeter sur l'autre sa propre faute, c'était remettre à sa place. Je t'ai répondu qu'en général les hommes supportaient mal d'obéir quand ils croyaient punir, je t'ai dit que les femmes avaient leurs façons retorses de parvenir à leurs fins en feignant d'être eues. (Arcan, 2004, p. 150)

L'ironie ici est très marquée : autant dans le procédé de transfert illocutoire des propos de l'amant rapportés que dans la réponse de la femme. L'homme, dominant, punit la femme, dominée, la remet à sa place par sa puissance éjaculatoire. Comme réponse à cette déclaration phallocrate, le personnage Nelly Arcan énonce explicitement les stratégies d'ironie que, selon elle, les femmes pratiquent sur leur partenaire masculin : dire et faire le contraire de ce que l'on pense pour arriver à ses fins. 
Arcan se moque également de son ancien amoureux à cause de ses aspirations artistiques d'apprenti écrivain et de son comportement colonialiste :

Devenir écrivain était pour toi un vieux rêve et pour moi, l'aboutissement de mon asocialité. En écrivant dans les cafés, tu voulais entretenir l'image de l'écrivain vu en train d'écrire, tu voulais écrire dans la pensée du livre dont on aurait suivi toutes les étapes et auquel on pourrait associer l'air tourmenté et traversé par le doute de l'écrivain penché sur son clavier, son air usé par la tâche de dire, son air de page blanche en attente de l'œuvre. Un jour, tu m'as dit que, lors des prises de photos promotionnelles pour ton futur roman, tu fumerais une cigarette; tu trouvais que les écrivains devaient s'opposer dans l'image à toutes les formes de propagande et surtout à celle des baby-boomers de la côte Ouest américaine. (Arcan, 2004, p. 170)

La dernière partie de l'extrait cité semble reprendre textuellement les mots de l'homme français, si bien que le transfert illocutoire est ici explicite. La description de l'amant dans les cafés en train d'écrire est la complète opposition entre le génie littéraire tourmenté qu'il aimerait devenir et l'écrivain narcissique, obsédé par son apparence qu'il semble plutôt être. Arcan utilise le langage de l'autre pour montrer le dérisoire de sa «pratique » d'écrivain : les expressions éculées et ampoulées comme «l'air tourmenté », "son air usé par la tâche de dire » ou encore "son air de page blanche en attente de l'œuvre » singent hyperboliquement et ridiculisent les aspirations artistiques risibles du Français. De plus, l'amant pense déjà à son image médiatique d'écrivain pseudo-révolutionnaire avant même d'avoir publié son roman : c'est donc l'apparence qui prend ici encore le pas sur le travail créateur. Sous le regard d'une auteure asociale et publiée dont le livre s'est bien vendu 
en France, le snobisme de l'apprenti écrivain est tourné en ridicule.

Arcan dénonce effectivement le comportement et le propos de certains hommes grâce à ce personnage du journaliste, mais le transfert illocutoire ironique a également à voir avec l'introspection. L'amant français est comme le reflet de l'auteure-narratrice-personnage dans le miroir; il est l'exact opposé, le contraire, mais un contraire qui attire follement, qui fascine: «Devant le mur de miroirs à Nova, [...] [r]ien ne pouvait plus m'atteindre, pas même ta voix, tu ne pouvais rien contre la plus grande obsession de ma vie qui est aussi la plus redoutable parce que je n'ai jamais trouvé la porte de sortie: mon reflet dans le miroir. » (Arcan, 2004, p. 157) Arcan détaille les défauts de ses traits, de son grain de peau, de ses cernes et de ses rides dans le miroir comme elle constate, en se comparant à l'amant français, son accent québécois, sa honte de colonisée, sa condition de femme aliénée. Le transfert illocutoire ironique écorche certes l'amant en dévoilant ses tendances misogynes et violentes, mais Arcan n'hésite cependant pas à l'élever au rang de l'Autre, meilleur et dominant.

\section{Auto-ironie}

Comme énoncé plus haut, Nelly Arcan n'épargne pas sa propre personne de son jugement corrosif. Elle pratique ce que Joubert appelle l'« auto-ironie » :

L'auto-ironie exclut toute velléité de se ménager soi-même comme cible. [...] [Elle] se définit donc avant tout par la spécificité de la cible de l'ironie, soit l'ironiste elle-même. Les auteures proposent des personnages qui s'évaluent sévèrement 
[...] ; ces «je » scrutent leurs moindres défauts et se servent de l'ironie pour transmettre leur tyrannique lucidité. (1998, p. 46)

Nelly Arcan se révèle extrêmement sévère envers le rôle déterminé par la condition des femmes. Joubert appelle cette auto-ironie "collective», celle qui englobe, à travers un personnage ou une situation, toutes les femmes qui partagent les mêmes fonctions convenues (1998, p. 46-47). Par exemple, Arcan écrit :

J'ai également connu les filles du Net stockées en masse dans ton ordinateur et qui, celles-là, portaient tous les noms regroupés en grandes catégories, les Schoolgirls, les College Girls et les Girls Nextdoor, les Wild Girlfriends et [...] les Fuckmeboots. Grâce à toi, j'ai appris que sur le Net il y avait peu de Women. (Arcan, 2004, p. 19)

Arcan énumère ici des catégories stéréotypées de « filles ». Fille comme dans «fille publique», prostituée, enfant jamais adulte, incapable de choisir pour elle-même. L'auteure ironise ainsi sur les femmes en général qui, comme elle, peinent à s'extirper du statut d'objet, particulièrement dans la sphère de la sexualité et de la pornographie. Cette auto-ironie se moque des stéréotypes et des topoï sexistes entretenus par la société en général et entérinés en partie par de nombreuses femmes. Également, la narratrice attaque ceux qui les nomment ainsi, c'est-à-dire la société qui ne veut pas de Women, c'est-àdire les consommateurs de pornographie qui préfèrent infantiliser et discréditer les femmes. Dans un seul énoncé, la cible de l'ironie d'Arcan peut être multiple. Toujours dans la perspective de l'auto-ironie collective, Arcan utilise des termes vulgaires («salope», «pute», « chatte») pour décrire les femmes. Par ailleurs, elle titre son roman Folle, reprenant ainsi l'insulte sexiste dont on a affublé les femmes de tout temps. 
Cette réappropriation des insultes est un procédé utilisé depuis longtemps par les groupes féministes. En utilisant le langage des hommes, les féministes détournent le sens de l'injure et contrefont le discours prépondérant. Ce qui est perçu comme dégradant dans la bouche du dominant devient ici une prise de pouvoir. Lucie Joubert voit là l'ironie au féminin à son meilleur : «Reprenant la vulgarité à leur compte, [les auteures] en annulent le potentiel dévalorisant et l'asservissent. C'est, à échelle réduite, une illustration du fonctionnement de toute leur ironie: renverser le sens, pour montrer jusqu'où elles peuvent aller...» (1998, p. 45)

L'auto-ironie d'Arcan attaquera également sa propre personne : elle pratiquera ce que Joubert appelle l'auto-ironie individuelle. Arcan ironise sur son propre désespoir après la rupture amoureuse. Loque humaine, elle passe ses journées à écouter des téléséries dans son appartement et n'en sort que pour aller louer les saisons suivantes :

Pendant ces trois mois j'ai payé plus de deux cents dollars de retard sur les locations, là-bas les employés étaient morts de rire dans mon dos. Chaque fois qu'ils me voyaient arriver, avec les locations de la veille dans les mains, ils se tenaient entre eux du coin de l'œil ou ils souriaient les yeux par terre quand leurs collègues se trouvaient trop loin pour la complicité. Ils avaient tous leur façon de se payer ma gueule sans me faire face, à la Boîte Noire, les employés ont du goût, ils aiment le cinéma de répertoire. (Arcan, 2004, p. 86)

La Boîte noire est un club vidéo de répertoire situé sur le Plateau-Mont-Royal. Lorsque la narratrice suppose aux employés un bon goût et un amour du cinéma de répertoire, elle instaure un effet de contraste avec la situation précédemment énoncée, lorsque les employés se moquent de son chagrin avec condescendance et absence de compassion. C'est le snobisme des 
intellectuels devant un cinéma populaire, le snobisme des inconnus devant la fameuse Nelly Arcan, auteure à succès, complètement dévastée par un chagrin d'amour.

Malgré tout, Lucie Joubert a tendance à voir dans l'autoironie individuelle un indice de santé :

Les narratrices qui recourent à l'auto-ironie semblent se dire : «Comment ai-je pu tomber si bas? » Chaque trait ironique qu'elles décochent dans leur propre direction annonce, d'une certaine façon, qu'on ne les y reprendra pas. À la fois constat d'une incongruité et d'un appel au changement, l'ironie sur soi [...] reste empreinte d'une empathie, quelquefois désillusionnée, pour le sujet (elles-mêmes) dont elles se moquent. (Joubert, 1998, p. 50-51)

À plusieurs égards, on peut être d'accord avec Joubert. Arcan demande bel et bien "Comment ai-je pu tomber si bas? » au lecteur, à l'amant français et à elle-même. Cependant, bien que d'une "lucidité tyrannique», pour reprendre l'expression de Joubert, Arcan ne fait pas appel au changement. Embourbée dans les fonctions convenues qu'elle dénonce (la putain, la folle, la femme amoureuse) et les propos paternalistes et hautement misogynes tenus par l'amant français, elle n'est pas l'agent de changement. Elle se laisse aller au nihilisme et au fatalisme jusqu'au suicide, destin choisi par Nelly Arcan adolescente, quinze ans plus tôt. On ne reprendra plus la jeune femme à tomber aussi bas, mais bien parce qu'Arcan-personnage préférera s'enlever la vie, suicider l'ironiste en elle.

\section{Renversement des inégalités au sein du couple hétérosexuel}

Notre hypothèse de départ veut que les outils langagiers et stylistiques de la méchanceté utilisés par Nelly Arcan dans Folle 
servent à renverser les inégalités au sein du couple hétérosexuel. L'humour en général et l'ironie en particulier, dans le couple hétérosexuel, permettent aux femmes de subvertir les rapports de pouvoir, selon Caroline Henchoz (2012). L'humour au féminin peut avoir plusieurs fonctions, que l'on peut rapporter à la tonalité ironique qu'utilise Arcan. On peut constater qu'il permet aux femmes de se distancier d'une situation autrement intolérable. Par exemple, dans Folle, la narratrice subit un avortement après avoir été quittée par l'homme qu'elle aime. Elle écrit: "Je suppose que dans un moment d'inattention, tu as dû te vider dans ma chatte au moins une fois. Je me demande si le besoin de voir son sperme a un lien avec le besoin de voir sa morve à l'intérieur des mouchoirs ou encore sa merde avant de vider la cuvette.» (Arcan, 2004, p. 68) Ici, le cynisme d'Arcan lui permet de relativiser, voire de ridiculiser l'acte de conception. Sa grossesse est ainsi considérée comme le simple fruit du hasard. Le personnage peut alors plus aisément se distancier de l'avortement et du choc émotif et physique qui en découle. Elle compare l'éjaculation de l'homme à un mouchoir souillé. Ironiquement, l'homme et la femme sont sur le même pied d'égalité dans la gestion de leurs déchets organiques. Le sperme est essuyé dans un mouchoir, le fœtus est aspiré chirurgicalement. On se débarrasse ensuite des deux sans grande émotion. On peut également relever l'utilisation d'un langage grossier dans le dernier extrait cité. À la vulgarité qu'Arcan dénonce dans les pratiques sexuelles de son ancien amoureux, elle répond par la vulgarité. Elle donne la monnaie de sa pièce à l'homme qui l'a abandonnée.

Caroline Henchoz donne aussi à l'humour chez la femme hétérosexuelle la fonction de critique des normes sociales et de 
reconnaissance du pouvoir social masculin (2012, p.91), ce qu'atteste effectivement le récit ironique d'Arcan. Par exemple, la narratrice s'insurge contre les différences biologiques entre les hommes et les femmes qui façonnent certains comportements sociaux :

Je disais qu'avoir des ovules qui circulaient de tous côtés de soi sans l'intervention de la volonté était une injustice impardonnable qui avait tracé le cours de l'histoire. Je disais que si la reproduction avait dépendu du plaisir des femmes, les femmes n'auraient eu d'autre choix que d'élargir à l'infini leur cercle d'hommes qui auraient alors vécu en se traînant à leurs pieds, les hommes auraient fondé leurs institutions par le bas et se seraient punis entre eux de leur incompétence. (Arcan, 2004, p. 84)

L'inversion des systèmes biologiques met ici en lumière une " injustice impardonnable » entre le statut de l'homme et celui de la femme. La satire des mœurs inversées rend patents les rapports inégaux entre les hommes et les femmes dans plusieurs sphères: sexuelle et amoureuse (reproduction, anatomie, orgasme, nombre de partenaires) et professionnelle et sociale (institutions, incompétence, punition). C'est aussi une inversion très cynique : on rit à imaginer les hommes consulter compulsivement les magazines pour apprendre à faire jouir leur femme, celle-ci en parfait contrôle de sa reproduction. On rit à les imaginer s'apprêter pour faire partie du « cercle infini » des femmes, jusqu'à ce qu'on y perçoive la critique cinglante de l'actuel comportement des femmes occidentales. C'est un rire, jaune, qui glace le sang de la lectrice féministe.

Parallèlement, il est important de préciser que le couple formé par Arcan et l'amant journaliste repose également sur l’opposition entre le Français et la Québécoise. Les conventions 
sociales du colonisateur et du colonisé sont ici pour Arcan source de raillerie.

Par exemple, parlant de leur première rencontre dans un rave, la narratrice explique : «Si je ne t'ai pas bien entendu ce soir-là, c'est parce que ton accent couvrait ton discours. » (Arcan, 2004, p. 32) L'effet ironique repose sur l'opposition entre « accent » et « discours ». Alors que l'accent est sonore, le discours est plutôt sémantique. Il est donc impossible, logiquement, qu'un accent couvre une idée. L'accent est plutôt la représentation métonymique du Français colonisateur, celui dont la culture, l'éducation, le parler, les mœurs sont meilleurs que ceux des colons. Par contre, l'accent, représentatif de la supériorité française sur les Québécois, ne sert qu'à couvrir des idées tordues sur les relations homme-femme, sur la sexualité et sur la pornographie. La domination de l'amant français n'est alors que dans le paraître, ou plutôt dans l'entendre. Notons toutefois qu'Arcan ne s'épargne pas non plus dans cet extrait. Elle remplit à merveille le rôle de la colonisée, celle obnubilée par le subterfuge du colonisateur.

L'article de Caroline Henchoz conclut que l'humour au féminin «n'est pas seulement l'indicateur du changement vers plus de symétrie conjugale, c'est aussi l'instrument de ce changement » (2012, p. 99). Cependant, rien n'est moins certain après la lecture de Folle. Certes, l'amant et plusieurs conventions sociales sont raillés, mais il reste que la plus grande victime de l'ironie d'Arcan est Arcan elle-même. Évidemment, elle s'évertue à décortiquer dans ses moindres détails la relation malsaine qu'elle entretenait avec un écrivain raté, dépendant à la pornographie, sadique et prétentieux, mais la grande perdante de cet échec amoureux, c'est la femme 
quittée et désespérée, qui écrit une lettre d'amour comme on envoie une bouteille à la mer. Si elle nomme les défauts de son amant comme causes de rupture, elle rejette néanmoins le tort sur elle-même. Par exemple, elle écrit :

Souvent au lit tu m'attachais et tu me prenais dans le manque de préparation pour me donner l'aspect d'une femme en lutte, parfois tu me frappais en dosant tes élans et souvent, le plaisir me tirait hors du corps. [...] Au début tu m'aimais entre autres pour ma souplesse, ensuite tu t'es lassé de moi, entre autres à cause de ma souplesse. (Arcan, 2004, p. 176)

Le chiasme à la dernière phrase a ici l'effet de renforcer l'opposition entre le début de la relation et la fin de celle-ci. L'effet ironique repose sur la polysémie du mot "souplesse ». La scène sexuelle évoquée juste avant peut laisser croire que la narratrice parle de la souplesse physique. Cependant, à la répétition du mot «souplesse » comme une cause de lassitude chez l'amant, on comprend que c'est aussi celle de l'adaptation, de plier l'échine, de se mettre à genoux. L'amant est blasé par sa maîtresse trop soumise, qui prend plaisir au sadomasochisme et qui en redemande. Arcan est d'une lucidité tyrannique et laconique: «Il paraît que pour s'attacher les hommes solidement, les femmes doivent montrer de la résistance.» (Arcan, 2004, p. 29)

En somme, la méchanceté de Nelly Arcan dans Folle repose sur la fictionnalisation de personnes réelles. Le personnage de l'amant français est sévèrement jugé et ridiculisé avec une ironie mordante. Ses aspirations professionnelles, ses dépendances, ses vices, ses défauts sont la cible des railleries de la narratrice. Plusieurs stratégies stylistiques (l'auto-ironie collective et individuelle, la réappropriation d'injures, le transfert illocutoire, etc.) sont 
utilisées à cet égard. L'ironie et la méchanceté dénoncent et renversent les inégalités au sein du couple hétérosexuel, formé par un Français et une Québécoise, mais nous sommes bien obligés de convenir que ces procédés littéraires échouent à faire de Nelly Arcan-personnage-narratrice-auteure l'agente de changements. L'ironie mène la narratrice au nihilisme et au fatalisme, ce qui a néanmoins pour effet de faire intervenir une autre sorte d'ironie, l'ironie du sort. Est-ce que l'ironie du sort pourrait quant à elle être considérée comme une prise de conscience et une invitation à la relativisation ? Encore là, rien n'est moins sûr. Il faudrait alors de nouveau considérer les implications autofictionnelles de l'ironie du sort dans la biographie d'une auteure comme Nelly Arcan, celle qui a fait de sa vie réelle et de sa mort un roman.

\section{Bibliographie}

ArCan, Nelly. (2004), Folle, Paris, Seuil.

BAUDELLE, Yves. (2007), «Autofiction et roman autobiographique: incidents de frontière », dans Robert Dion et al. (dir.), Vies en récit. Formes littéraires et médiatiques de la biographie et de l'autobiographie, Québec, Nota bene, p. 43-70.

HAREL, Simon. (2010), Attention écrivains méchants, Québec, Les Presses de l'Université Laval.

HENCHOZ, Caroline. (2012), «De l'humour comme d'une compétence sociale pour gérer et contester les rapports de pouvoir et les inégalités dans le couple», Recherches féministes, vol. 25, $\mathrm{n}^{\circ} 2$, p. 83-102. 
JOUBERT, Lucie. (1998), Le Carquois de velours: l'ironie au féminin dans la littérature québécoise (1960-1980), Montréal, L'Hexagone.

OUELLETTE-MichalSKA, Madeleine. (2007), Autofiction et dévoilement de soi, Montréal, XYZ.

RiTouX, Nicolas. (2006), «Isabelle», Urbania, vol. 13, p. 30-31.

\section{Résumé}

Dans Folle (2004), Nelly Arcan prête à un personnage les traits d'un ancien amoureux : l'auteure rend ainsi publics les vices et les défauts de son ancien amant. La méchanceté d'Arcan est intrinsèquement liée au désir de vengeance du dominé humilié. Par contre, sa hargne ne s'arrête pas là : l'ironie d'Arcan écorche aussi son alter ego fictif. Elle se prend comme cible pour montrer les inégalités au sein du couple hétérosexuel et utilise l'auto-ironie afin de renverser les codes de domination entre les hommes et les femmes, les Français et les Québécois.

\section{Abstract}

In the story Folle (2004), Nelly Arcan lent the features of a former lover of hers to a fictional character: thereby the author exposes his vices and his flaws to the public. Arcan's wickedness is tied to the desire for revenge of the dominated one. But her aggression will not stop there: Arcan's irony also lashes at her fictional alter ego. She targets herself to show the inequalities within a heterosexual relationship and uses selfirony to interchange the domination codes between men and women, French and Quebecers. 